\title{
MEROMORPHIC MULTIVALENT CLOSE-TO-CONVEX FUNCTIONS
}

\author{
A. E. LIVINGSTON( $\left.{ }^{1}\right)$
}

1. Introduction. Recently the author [5] has discussed some properties of the class of $p$-valent regular close-to-convex functions, called $\mathscr{K}(p)$. It is the purpose of this paper to generalize some of these results to the meromorphic case.

Let $f(z)$ be meromorphic for $|z|<1$ with $q(1 \leqq q \leqq p)$ poles at the origin and $f(z) \neq 0$ for $|z|<1$. We shall say that $f(z)$ is in $S_{1}^{*}(p)$ if there exists a $\rho(0<\rho<1)$ such that for $z=r e^{i \theta}(\rho<r<1)$,

$$
\operatorname{Re}\left[\frac{z f^{\prime}(z)}{f(z)}\right]<0
$$

and

$$
\int_{0}^{2 \pi} d \arg f(z)=\int_{0}^{2 \pi} \operatorname{Re}\left[\frac{z f^{\prime}(z)}{f(z)}\right] d \theta=-2 p \pi .
$$

We shall say that $f(z)$ is in $S_{2}^{*}(p)$ if it is regular on $|z|=1$ and if (1.1) and (1.2) hold for $|z|=1$. If $f(z)$ is in $S_{1}^{*}(p)$, there exists a $\delta(0<\delta<1)$ such that $f(r z)$ is in $S_{2}^{*}(p)$ if $\delta<r<1$.

We set $S^{*}(p)=S_{1}^{*}(p) \cup S_{2}^{*}(p)$ and say that a function in $S^{*}(p)$ is starlike of order $p$.

Condition (1.2) along with the argument principle implies that a function in $S^{*}(p)$ has exactly $p$ poles in $|z|<1$. It is easily seen that a function $f(z)$, meromorphic in $|z|<1$, is in $S^{*}(p)$ if and only if the function $[f(z)]^{-1}$ is regular and $p$-valently starlike in $|z|<1$. Since the reciprocal of a $p$-valent function is $p$ valent, a function in $S^{*}(p)$ is $p$-valent in $|z|<1$. Also, using the fact that a regular $p$-valent starlike function can be written as the $p$ th power of a regular univalent starlike function, it is easily seen that a function in $S^{*}(p)$ with $p$ poles at the origin can be written as the $p$ th power of a meromorphic univalent starlike function.

Let $F(z)$ be meromorphic in $|z|<1$ with $q(1 \leqq q \leqq p)$ poles at the origin and with at most $p$ poles in $|z|<1$. We shall say that $F(z)$ is in $\mathscr{K}_{1}^{*}(p)$ if there exists a function in $S^{*}(p)$ and a $\rho(0<\rho<1)$ such that for $z=r e^{i \theta}(\rho<r<1)$

$$
\operatorname{Re}\left[\frac{z F^{\prime}(z)}{f(z)}\right]>0 \text {. }
$$

Received by the editors July 8, 1964, and, in revised form, November 18, 1964.

(1) This paper is part of a Thesis written under the direction of Professor M. S. Robertson at Rutgers University. 
We shall say that $F(z)$ is in $\mathscr{K}_{2}^{*}(p)$ if $F(z)$ is regular on $|z|=1$ and if there exists a function $f(z)$ in $S_{2}^{*}(p)$ such that $(1.3)$ is satisfied for $|z|=1$. If $F(z)$ is in $\mathscr{K}_{1}^{*}(p)$, there exists a $\delta(0<\delta<1)$ such that $F(r z)$ is in $\mathscr{K}_{2}^{*}(p)$ if $\delta<r<1$.

We set $\mathscr{K}^{*}(p)=\mathscr{K}_{1}^{*}(p) \cup \mathscr{K}_{2}^{*}(p)$ and say that a function in $\mathscr{K}^{*}(p)$ is closeto-convex of order $p$.

The class $\mathscr{K}^{*}(1)$ was defined by Libera and Robertson [4] and Pommerenke [7]. It was shown in both papers that a function in $\mathscr{K}^{*}(1)$ need not be univalent. To show that a function in $\mathscr{K}^{*}(p)$ need not be $p$-valent, let $F(z)$ be such that

$$
\frac{z F^{\prime}(z)}{z^{-p}}=\frac{1+z^{2 p}}{1-z^{2 p}} \quad(|z|<1) \text {. }
$$

Then

$$
F(z)=-\frac{1}{p z^{p}}+\frac{2}{p} z^{2 p}+\frac{2}{3 p} z^{3 p}+\cdots \quad(0<|z|<1) .
$$

If $F(z)$ was $p$-valent, then

$$
F\left(z^{1 / p}\right)=-\frac{1}{p z}+\frac{2}{p} z+\cdots \quad(0<|z|<1)
$$

would be univalent, and so would

$$
-p F\left(z^{1 / p}\right)=\frac{1}{z}-2 z+\cdots \quad(0<|z|<1) .
$$

But this is impossible, since the coefficient of $z$ has modulus greater than 1. Thus $F(z)$ is at least $2 p$-valent.

Necessary and sufficient conditions for a function to be in $\mathscr{K}^{*}(1)$ have been given in [4] and [7]. In $\$ 2$ we obtain necessary conditions for a function $F(z)$ to be in $\mathscr{K}^{*}(p)$ and show that these conditions with the added assumptions of regularity on $|z|=1$ and $F^{\prime}(z) \neq 0$ in $|z| \leqq 1$ are sufficient.

Recently, Royster [8] has shown that if

$$
f(z)=\sum_{n=-p}^{\infty} a_{n} z^{n} \quad(0<|z|<1)
$$

is in $S^{*}(p)$ then $\left|a_{n}\right|=O(1 / n)$. In $\S 3$ we will extend this result to functions in $\mathscr{K}^{*}(p)$ with $p$ poles at the origin. This result was obtained for $\mathscr{K}^{*}(1)$ by Libera and Robertson [4] and Pommerenke [7].

2. The class $\mathscr{K}^{*}(p)$.

THEOREM 1. If $F(z)$ is in $\mathscr{K}^{*}(p)$, then there exists $\rho(0<\rho<1)$ such that for $z=r e^{i \theta}(\rho<r<1)$ 


$$
\int_{0}^{2 \pi} d \arg d F(z)=\int_{0}^{2 \pi} \frac{d}{d \theta} \arg \left[r e^{i \theta} F^{\prime}\left(r e^{i \theta}\right)\right] d \theta=-2 p \pi
$$

and for any $\theta_{1}$ and $\theta_{2}$ with $0 \leqq \theta_{1}<\theta_{2} \leqq 2 \pi$

$$
\int_{\theta_{1}}^{\theta_{2}} d \arg d F(z)<\pi \text {. }
$$

Proof. Suppose $F(z)$ is in $\mathscr{K}_{1}^{*}(p)$. Then there exists $f(z)$ in $S^{*}(p)$ and $\rho$ $(0<\rho<1)$ such that (1.1), (1.2) and (1.3) hold for $\rho<|z|<1$.

Since $\operatorname{Re}\left[z F^{\prime}(z) / f(z)\right]>0$ for $|z|=r(\rho<r<1)$, we may define

$$
\arg \left[z F^{\prime}(z) / f(z)\right]
$$

to be single valued and continuous for $|z|=r$ and such that

$$
\left|\arg \frac{z F^{\prime}(z)}{f(z)}\right|<\frac{\pi}{2} \quad(|z|=r) .
$$

Furthermore, since $z F^{\prime}(z) \neq 0$ for $|z|=r$, we may define arg $\left[z F^{\prime}(z)\right]$ to be single valued and continuous for $|z|=r$. Since $f(z)=\left[f(z) / z F^{\prime}(z)\right]\left[z F^{\prime}(z)\right]$, we may define $\arg [f(z)]=\arg \left[z F^{\prime}(z)\right]-\arg \left[z F^{\prime}(z) / f(z)\right]$ to be a single valued and continuous determination of $\arg [f(z)]$ for $|z|=r$. Then

$$
\left|\arg z F^{\prime}(z)-\arg f(z)\right|=\left|\arg \frac{z F^{\prime}(z)}{f(z)}\right|<\frac{\pi}{2} \quad(|z|=r) .
$$

It is easily seen that (2.4) implies

$$
-\pi+\int_{\theta_{1}}^{\theta_{2}} d \arg f(z)<\int_{\theta_{1}}^{\theta_{2}} d \arg d F(z)<\pi+\int_{\theta_{1}}^{\theta_{2}} d \arg f(z)
$$

for $\theta_{1}<\theta_{2}$ and $|z|=r$. Since $f(z)$ is in $S^{*}(p)$,

$$
\int_{\theta_{1}}^{\theta_{2}} d \arg f(z)<0 \quad(|z|=r) .
$$

Thus we obtain (2.2) for $|z|=r$ from the right side of (2.5). Letting $\theta_{1}=0$ and $\theta_{2}=2 \pi$ in (2.5) and noting that

we obtain

$$
\int_{0}^{2 \pi} d \arg f(z)=-2 p \pi
$$

$$
-(2 p+1) \pi<\int_{0}^{2 \pi} d \arg d F(z)<-(2 p-1) \pi .
$$

However, the integral appearing in (2.6) is an integral multiple of $2 \pi$. Thus (2.1) holds for $|z|=r$. Since $r$ was arbitrary $(\rho<r<1)(2.1)$ and (2.2) hold for $\rho<|z|<1$. 
If $F(z)$ is in $\mathscr{K}_{2}^{*}(p)$, then the preceding argument with $r=1$ shows that (2.1) and (2.2) hold for $|z|=1$. But since $F(z)$ is regular near $|z|=1$, we can show the existence of a $\rho(0<\rho<1)$ such that (2.1) and (2.2) hold for $\rho<|z| \leqq 1$.

Using (2.1) and the argument principle we immediately obtain the following corollary.

Corollary 1. If $F(z)$ is in $\mathscr{K}^{*}(p)$, then $F^{\prime}(z)$ has at least $(p+1)$ poles in $|z|<1$ and if $F^{\prime}(z) \neq 0$ for $|z|<1$, then $F^{\prime}(z)$ has exactly $(p+1)$ poles in

THEOREM 2. Let $F(z)$ be meromorphic in $|z|<1$ with $q(1 \leqq q \leqq p)$ poles at the origin. If $F^{\prime}(z) \neq 0$ for $0<|z| \leqq 1$ and $F(z)$ is regular on $|z|=1$ and if (2.1) and (2.2) hold for $|z|=1$, then $F(z)$ is in $\mathscr{K}_{2}^{*}(p)$.

Proof. Consider the function $G(z)$, regular for $|z|=1$, given by

$$
G(z)=\int_{0}^{z} \frac{d z}{z^{2} F^{\prime}(z)}=b_{q} z^{q}+\cdots .
$$

Since $z F^{\prime}(z) \neq 0$ for $|z|=1$ we may define $\arg \left[z F^{\prime}(z)\right]$ to be single valued and continuous for $|z|=1$. Since $z G^{\prime}(z)=\left[z F^{\prime}(z)\right]^{-1}$, we may define $\arg z G^{\prime}(z)$ $=-\arg z F^{\prime}(z)$. Thus, for $|z|=1$

$$
\int_{0}^{2 \pi} d \arg d G(z)=2 p \pi
$$

and

$$
\int_{\theta_{1}}^{\theta_{2}} d \arg d G(z)>-\pi \quad\left(\theta_{1}<\theta_{2}\right) .
$$

The author has shown (Theorem $3[5])$ that under these conditions $G(z)$ is in $\mathscr{K}(p)$. That is, there exists $g(z)$, regular for $|z| \leqq 1$ such that

$$
\operatorname{Re}\left[\frac{z g^{\prime}(z)}{g(z)}\right]>0 \quad(|z|=1)
$$

and

$$
\operatorname{Re}\left[\frac{z G^{\prime}(z)}{g(z)}\right]>0 \quad(|z|=1) .
$$

The function $f(z)=[g(z)]^{-1}$ is in $S^{*}(p)$ and

$$
\frac{z G^{\prime}(z)}{g(z)}=z G^{\prime}(z) f(z)=\frac{f(z)}{z F^{\prime}(z)} .
$$

Thus 


$$
\operatorname{Re}\left[\frac{z F^{\prime}(z)}{f(z)}\right]=\operatorname{Re}\left[\frac{g(z)}{z G^{\prime}(z)}\right]>0 \quad(|z|=1) .
$$

Therefore $F(z)$ is in $\mathscr{K}_{2}^{*}(p)$.

Using the same procedure as above and by appealing to Theorem 2 [5], we may remove the condition of regularity on $|z|=1$, if $q=p$. We thus have the following theorem.

THEOREM 3. Let

$$
F(z)=\sum_{n=-p}^{\infty} a_{n} z^{n} \quad(0<|z|<1)
$$

be meromorphic for $|z|<1$ and $F^{\prime}(z) \neq 0$. If there exists a $\rho(0<\rho<1)$ such that (2.1) and (2.2) hold for $\rho<|z|<1$, then $F(z)$ is in $\mathscr{K}^{*}(p)$.

We will have need of the next lemma in what follows.

Lemma 1. Let $F(z)$ be in $\mathscr{K}_{2}^{*}(p)$. Then, there exists a function

$$
f(z)=\sum_{n=-p}^{\infty} b_{n} z^{n} \quad(0<|z|<1)\left(\left|b_{-p}\right|=1\right),
$$

in $S_{2}^{*}(p)$, such that

$$
\operatorname{Re}\left[\frac{z F^{\prime}(z)}{f(z)}\right]>0 \quad(|z|=1) .
$$

Proof. There exists a function $g(z)$ in $S_{2}^{*}(p)$ with $s$ poles $(1 \leqq s \leqq p)$ at the origin such that,

$$
\operatorname{Re}\left[\frac{z F^{\prime}(z)}{g(z)}\right]>0 \quad(|z|=1) .
$$

The function $g(z)$ has $(p-s)$ nonzero poles in $|z|<1$. Let $\alpha_{1}, \alpha_{2}, \cdots, \alpha_{p-s}$ be these poles and let

and

$$
h(z)=z^{s-p} \prod_{i=1}^{p-s}\left(z-\alpha_{i}\right)\left(1-\bar{\alpha}_{i} z\right)
$$

$$
f(z)=h(z) g(z)=\sum_{n=-p}^{\infty} c_{n} z^{n} \quad(0<|z|<1) .
$$

Since $\left[z h^{\prime}(z) / h(z)\right]$ is purely imaginary on $|z|=1$ and $\operatorname{Re}\left[z g^{\prime}(z) / g(z)\right]<0$ for $|z|=1$, then $\operatorname{Re}\left[z f^{\prime}(z) / f(z)\right]<0$ for $|z|=1$. Furthermore, since $f(z)$ has $p$ poles in $|z| \leqq 1$, all of them at the origin, and since $f(z) \neq 0$ in $|z| \leqq 1$,

$$
\int_{0}^{2 \pi} \operatorname{Re}\left[\frac{e^{i \theta} f^{\prime}\left(e^{i \theta}\right)}{f\left(e^{i \theta}\right)}\right] d \theta=-2 p \pi
$$


Thus, $f(z)$ in $S_{2}^{*}(p)$. Furthermore,

$$
\frac{z F^{\prime}(z)}{f(z)}=\frac{z^{p-s} z F^{\prime}(z)}{\prod_{i=1}^{p-s}\left(z-\alpha_{i}\right)\left(1-\bar{\alpha}_{i} z\right) g(z)} .
$$

But $z^{p-s}\left[\prod_{i=1}^{p-s}\left(z-\alpha_{i}\right)\left(1-\bar{\alpha}_{i} z\right)\right]^{-1}$ is real and positive on $|z|=1$. Therefore

$$
\operatorname{Re}\left[\frac{z F^{\prime}(z)}{f(z)}\right]>0 \quad(|z|=1) .
$$

Replacing $f(z)$ by $1 /\left|c_{-p}\right| f(z)$, the proof of the lemma is completed.

THEOREM 4. If $F(z)$ is in $\mathscr{K}^{*}(p)$ and has all its poles at the origin, then necessarily it has $p$ poles there and $F^{\prime}(z) \neq 0$ for $|z|<1$.

Proof. Suppose

$$
F(z)=\sum_{n=-q}^{\infty} a_{n} z^{n} \quad(0<|z|<1)(1 \leqq q \leqq p) .
$$

There exists a $\rho(0<\rho<1)$ such that $F(r z)$ is in $\mathscr{K}_{2}^{*}(p)$ if $\rho<r<1$. By Lemma 1 , there exists

$$
f(z)=\sum_{n=-p}^{\infty} b_{n} z^{n} \quad(0<|z|<1)
$$

in $S_{2}^{*}(p)$ such that

$$
\operatorname{Re}\left[\frac{r z F^{\prime}(r z)}{f(z)}\right]>0 \quad(|z|=1) .
$$

Since $f(z) \neq 0$ for $|z| \leqq 1$,

$$
\frac{r z F^{\prime}(r z)}{f(z)}=\sum_{n=p-q}^{\infty} c_{n} z^{n}
$$

is regular for $|z| \leqq 1$. Thus,

$$
\operatorname{Re}\left[\frac{r z F^{\prime}(r z)}{f(z)}\right]>0 \quad(|z| \leqq 1)
$$

Therefore, we must necessarily have $q=p$ and $F^{\prime}(r z) \neq 0$ for $|z| \leqq 1$. Thus, $F^{\prime}(z) \neq 0$ for $|z| \leqq r$. Since $r$ was arbitrary $(\rho<r<1), F^{\prime}(z) \neq 0$ for $|z|<1$.

If $F(z)$ has all its poles at the origin we may improve Lemma 1 by removing the condition of regularity on $|z|=1$.

LEMMA 2. Let

$$
F(z)=\sum_{n=-p}^{\infty} a_{n} z^{n} \quad(0<|z|<1)
$$


be in $\mathscr{K}^{*}(p)$. Then there exists

$$
f(z)=\sum_{n=-p}^{\infty} b_{n} z^{n} \quad(0<|z|<1) \quad\left(\left|b_{-p}\right|=1\right)
$$

in $S^{*}(p)$ such that

$$
\operatorname{Re} \frac{z F^{\prime}(z)}{f(z)}>0 \quad(|z|<1) .
$$

Proof. There exists a $\rho(0<\rho<1)$ such that the function $F_{r}(z)=F(r z)$ is in $\mathscr{K}_{2}^{*}(p)$ if $\rho<r<1$. Then by Lemma 1 there exists

$$
f_{r}(z)=\sum_{n=-p}^{\infty} c_{n} z^{n} \quad(0<|z|<1)\left(\left|c_{-p}\right|=1\right)
$$

in $S_{2}^{*}(p)$, such that

$$
\operatorname{Re}\left[\frac{z F_{r}^{\prime}(z)}{f_{r}(z)}\right]>0 \quad(|z| \leqq 1)
$$

Let $r_{i}\left(\rho<r_{i}<1\right)$ be an increasing sequence tending to 1 . The functions $\left[f_{r_{i}}(z)\right]^{-1}$ are regular and $p$-valently starlike and have the moduli of their first $p$ coefficients fixed. The class of regular and $p$-valently starlike functions with the moduli of their first $p$ coefficients fixed forms a normal family of functions [1]. Thus, we can obtain a subsequence $\left[f_{r_{i_{k}}}(z)\right]^{-1}$ tending uniformly in every closed subset of $|z|<1$ to a function $f(z)$ regular and $p$-valently starlike and such that

$$
f(z)=\sum_{n=p}^{\infty} d_{n} z^{n} \quad(|z|<1)\left(\left|d_{p}\right|=1\right) .
$$

Since $F_{r_{i_{k}}}(z)$ tends to $F(z)$ as $r_{i_{k}}$ tends to 1 and since

$$
\operatorname{Re}\left[z F_{r_{i_{k}}}(z)\left[f_{r_{i_{k}}}(z)\right]^{-1}\right]>0 \quad \text { for }|z|<1
$$

we have

$$
\operatorname{Re}\left[z F^{\prime}(z) f(z)\right]>0 \quad \text { for }|z|<1
$$

But

$$
g(z)=[f(z)]^{-1}=\sum_{n=-p}^{\infty} b_{n} z^{n} \quad(0<|z|<1)\left(\left|b_{-p}\right|=1\right)
$$

is in $S^{*}(p)$ and

$$
\operatorname{Re}\left[\frac{z F^{\prime}(z)}{g(z)}\right]=\operatorname{Re}\left[z F^{\prime}(z) f(z)\right]>0 \quad \text { for }|z|<1 .
$$

3. The coefficients of a function in $\mathscr{K}^{*}(p)$. We will make use of the following lemma, proven by Royster [8] and the author [6]. 
LEMMA 3. Let

$$
f(z)=\sum_{n=-p}^{\infty} b_{n} z^{n} \quad(0<|z|<1) \quad\left(\left|b_{-p}\right|=1\right)
$$

be in $S^{*}(p)$, then for $n \geqq 1$

$$
\left|b_{n}\right| \leqq \frac{2 p}{(n+p) \sqrt{ } p}\left(\sum_{k=-p}^{-1}|k|\left|b_{k}\right|^{2}\right)^{1 / 2}
$$

The following lemma was proven for $p=1$ by Pommerenke [7].

LEMMA 4. Let

$$
F(z)=\frac{1}{z^{p}}+\sum_{n=-(p-1)}^{\infty} a_{n} z^{n} \text { and } f(z)=\frac{e^{i \beta}}{z^{p}}+\sum_{n=-(p-1)}^{\infty} b_{n} z^{n}, \quad(0<|z|<1)
$$

and let $U(z)=\operatorname{Re}\left[z F^{\prime}(z) / f(z)\right]$, then for $r<1$

$$
\begin{aligned}
n a_{n}= & -p e^{-i \beta} b_{n}+\frac{1}{\pi} \int_{0}^{2 \pi}\left[U\left(r e^{i \theta}\right) \frac{e^{-i n \theta}}{r^{n}}\right] \\
& \times\left[f\left(r e^{i \theta}\right)-\sum_{k=n}^{\infty} b_{k}\left(r e^{i \theta}\right)^{k}\right] d \theta .
\end{aligned}
$$

Proof. Let

$$
\frac{z F^{\prime}(z)}{f(z)}=-p e^{-i \beta}+\sum_{k=1}^{\infty} C_{k} z^{k} \quad(|z|<1)
$$

Then

$$
\begin{aligned}
\frac{-p}{z^{p}}+\sum_{n=-(p-1)}^{\infty} n a_{n} z^{n}= & {\left[-p e^{-i \beta}+\sum_{k=1}^{\infty} C_{k} z^{k}\right]\left[\frac{e^{i \beta}}{z^{p}}+\sum_{n=-(p-1)}^{\infty} b_{n} z^{n}\right] } \\
= & \frac{-p}{z^{p}}-p e^{-i \beta}\left[\sum_{n=-(p-1)}^{\infty} b_{n} z^{n}\right]+e^{i \beta}\left[\sum_{k=1}^{\infty} C_{k} z^{k-p}\right] \\
& +\sum_{n=-(p-2)}^{\infty}\left[\sum_{k=1}^{n+p-1} C_{k} b_{n-k} z^{n}\right.
\end{aligned}
$$

Thus, for $n \geqq 1$

$$
n a_{n}=-p e^{-i \beta} b_{n}+e^{i \beta} C_{p+n}+\sum_{k=1}^{n+p-1} C_{k} b_{n-k} .
$$

Now

$$
C_{k}=\frac{1}{r^{k} \pi} \int_{0}^{2 \pi} U\left(r e^{i \theta}\right) e^{-i k \theta} d \theta
$$


Substituting into (3.2), we obtain

$$
\begin{aligned}
n a_{n}= & -p e^{-i \beta} b_{n}+\frac{1}{\pi} \int_{0}^{2 \pi}\left[U\left(r e^{i \theta}\right) \frac{e^{-i n \theta}}{r^{n}}\right] \\
& \times\left[\frac{e^{i \beta}}{r^{p} e^{i p \theta}}+\sum_{k=1}^{n+p-1} r^{n-k} e^{i(n-k) \theta} b_{n-k}\right] d \theta \\
= & -p e^{-i \beta} b_{n}+\frac{1}{\pi} \int_{0}^{2 \pi}\left[U\left(r e^{i \theta}\right) \frac{e^{-i n \theta}}{r^{n}}\right] \\
& \times\left[f\left(r e^{i \theta}\right)-\sum_{k=n}^{\infty} b_{k}\left(r e^{i \theta}\right)^{k}\right] d \theta .
\end{aligned}
$$

TheOREM 5. Let

$$
F(z)=\sum_{n=-p}^{\infty} a_{n} z^{n} \quad(0<|z|<1)\left(a_{-p} \neq 0\right)
$$

be in $\mathscr{K}^{*}(p)$, then $\left|a_{n}\right|=O\left(n^{-1}\right)$.

Proof. We may assume without loss of generality that $a_{-p}=1$. There exists, by Lemma 2 ,

$$
f(z)=\frac{e^{i \beta}}{z^{p}}+\sum_{n=-(p-1)}^{\infty} b_{n} z^{n} \quad(0<|z|<1)
$$

in $S^{*}(p)$ such that

$$
\left[\operatorname{Re} \frac{z F^{\prime}(z)}{f(z)}\right]>0 \quad(|z|<1) .
$$

Let $U(z)=\operatorname{Re}\left[z F^{\prime}(z) / f(z)\right]$, then by a well-known result on harmonic functions,

$$
\frac{1}{\pi} \int_{0}^{2 \pi} U\left(r e^{i \theta}\right) d \theta=2 U(0)=-2 p \cos \beta \leqq 2 p .
$$

By Lemma 4 , we have for $n \geqq 1$

$$
\begin{aligned}
n\left|a_{n}\right| \leqq p\left|b_{n}\right|+\frac{1}{\pi r^{n}}\left|\int_{0}^{2 \pi} U\left(r e^{i \theta}\right) e^{-i n \theta} \sum_{k=n}^{\infty} b_{k}\left(r e^{i \theta}\right)^{k} d \theta\right| \\
+\frac{1}{\pi r^{n}}\left|\int_{0}^{2 \pi} U\left(r e^{i \theta}\right) f\left(r e^{i \theta}\right) e^{-i n \theta} d \theta\right| \\
\leqq p\left|b_{n}\right|+\frac{2 p}{r^{n}} \sum_{k=n}^{\infty}\left|b_{k}\right| r^{k}+\frac{1}{\pi r^{n}} \int_{0}^{2 \pi} U\left(r e^{i \theta}\right)\left|f\left(r e^{i \theta}\right)\right| d \theta
\end{aligned}
$$


The Area Theorems of Golusin [2] and Kobori [3] give for $n \geqq 1$

$$
\sum_{k=n}^{\infty} k\left|b_{k}\right|^{2} \leqq \sum_{k=1}^{\infty} k\left|b_{k}\right|^{2} \leqq \sum_{k=-p}^{-1}|k|\left|b_{k}\right|^{2}
$$

We thus have,

$$
\begin{aligned}
\frac{2 p}{r^{n}} \sum_{k=n}^{\infty}\left|b_{k}\right| r^{k} & \leqq \frac{2 p}{r^{n}}\left[\sum_{k=n}^{\infty} k\left|b_{k}\right|^{2}\right]^{1 / 2}\left[\sum_{k=n}^{\infty} \frac{r^{2 k}}{k}\right]^{1 / 2} \\
& \leqq \frac{2 p}{r^{n}}\left[\sum_{k=-p}^{-1}|k|\left|b_{k}\right|^{2}\right]^{1 / 2}\left[\frac{1}{n} \sum_{k=n}^{\infty} r^{2 k}\right]^{1 / 2} \\
& =2 p\left[\sum_{k=-p}^{-1}|k|\left|b_{k}\right|^{2}\right]^{1 / 2}\left[n\left(1-r^{2}\right)\right]^{-1 / 2}
\end{aligned}
$$

Also for $n \geqq p$, by Lemma 3

$$
\begin{aligned}
\left|b_{n}\right| & \leqq \frac{2 p}{(p+n) \sqrt{ } p}\left[\sum_{k=-p}^{-1}|k|\left|b_{k}\right|^{2}\right]^{1 / 2} \\
& \leqq \frac{1}{\sqrt{ } p}\left[\sum_{k=-p}^{-1}|k|\left|b_{k}\right|^{2}\right]^{1 / 2} .
\end{aligned}
$$

Since $[f(z)]^{-1}$ is $p$-valently star like we have

$$
\left|f\left(r e^{i \theta}\right)\right|^{-1} \geqq \frac{r^{p}}{1+r)^{2 p}}
$$

or

$$
\left|f\left(r e^{i \theta}\right)\right| \leqq \frac{(1+r)^{2 p}}{r^{p}}
$$

Therefore, for $n \geqq p$

$$
\begin{aligned}
\frac{1}{\pi r^{n}} \int_{0}^{2 \pi} U\left(r e^{i \theta}\right) & \left|f\left(r e^{i \theta}\right)\right| d \theta \\
& \leqq \frac{(1+r)^{2 p}}{r^{p+n}} \frac{1}{\pi} \int_{0}^{2 \pi} U\left(r e^{i \theta}\right) d \theta \\
& \leqq \frac{2 p(1+r)^{2 p}}{r^{p+n}} \leqq \frac{2 p 4^{p}}{r^{2 n}}
\end{aligned}
$$

From (3.3), (3.4), (3.5) and (3.6) we have for $n \geqq p$ and any $r<1$

$$
n\left|a_{n}\right| \leqq\left[\sqrt{ } p+2 p\left[n\left(1-r^{2}\right)\right]^{-1 / 2}\right]\left[\sum_{k=-p}^{-1}|k|\left|b_{k}\right|^{2}\right]^{1 / 2}+2 p 4^{p} r^{-2 n} \text {. }
$$


Let $r^{2}=(1-1 / n)$, then for $n \geqq p+1$

$$
\begin{aligned}
n\left|a_{n}\right| & \leqq(\sqrt{ } p+2 p)\left[\sum_{k=-p}^{-1}|k|\left|b_{k}\right|^{2}\right]^{1 / 2}+2 p 4^{p}(1+1 /(n-1))^{n} \\
& \leqq(\sqrt{ } p+2 p)\left[\sum_{k=-p}^{-1}|k|\left|b_{k}\right|^{2}\right]^{1 / 2}+2 p 4^{p} \frac{(p+1)}{p} e .
\end{aligned}
$$

Thus, $\left|a_{n}\right|=O\left(n^{-1}\right)$.

\section{REFERENCES}

1. M. Cartwright, Some inequalities in the theory of functions, Math. Ann.111 (1935),98-118.

2. G. M. Golusin, On p-valent functions, Mat. Sb. (N. S.) 8 (1940), 277-284.

3. A. Kobori, Zur Theorie der mehrwertigen Funktionen, Japan. J. Math. 19 (1947), 301-319.

4. R. J. Libera and M. S. Robertson, Meromorphic close-to-convex functions, Michigan Math. J. 8 (1961), 167-175.

5. A. E. Livingston, P-valent close-to-convex functions, Trans. Amer. Math. Soc. 115 (1965), 161-179.

6. - p-valent close-to-convex functions, Thesis, Rutgers, The State University, New Brunswick, N.J., 1963.

7. C. H. Pommerenke, Über einige klassen meromorpher schlichter Funktionen, Math. Z. 78, (1962), 263-284.

8. W. C. Royster, Meromorphic starlike multivalent functions, Trans. Amer. Math. Soc. 107 (1963), 300-308.

\section{LAFAyetTe College,}

Easton, Pennsyluania 